\title{
Isomeric Separation of N-Glycopeptides Derived from Glycoproteins by Porous Graphitic Carbon (PGC) LC-MS/MS
}

Rui Zhu ${ }^{\ddagger}$, Yifan Huang ${ }^{\ddagger}$ Jingfu Zhao, Jieqiang Zhong and Yehia Mechref*

Department of Chemistry and Biochemistry, Texas Tech University, Lubbock, TX

\section{*Corresponding Author}

Department of Chemistry and Biochemistry

Texas Tech University

Lubbock, TX 79409-1061

Email: yehia.mechref@ttu.edu

Tel: 806-742-3059

Fax: 806-742-1289

$\$$ Both authors contributed equally 
Table of Contents

Table S1. Retention times and theirs RSDs of tryptic digested fetuin glycopeptide isomers on LCPDCPLLAPL ${ }^{156}$ NDSR over 15 runs.

Table S2. Comparison of quantitative results of glycopeptides derived from RNaseB between PGC-LC-MS and previous ${ }^{1} \mathrm{H}$ NMR study by Fu et al. ${ }^{1}$

Table S3. Summary of the retention times and glycoforms for each glycopeptide isomers derived from AGP glycopeptides. AGP was digested with trypsin and chymotrypsin. See additional information provided in the method section of the paper.

Figure S1. EICs of peptides (a-d) and glycopeptides (e-f) in tryptic digested fetuin sample under acidic conditions. The highly sialylated structures were not eluted from the column.

Figure S2. EICs of peptides (a-d) and glycopeptides (e-f) in tryptic digested fetuin sample under basic conditions. Glycopeptides were isomerically separated with stronger retention and uniformed charge states. Deglycosylated peptide backbone were not resolved indicating the separation was prompted by glycan isoforms (g).

Figure S3. Intensity comparison of tryptic digest of bovine fetuin under (a) $100 \%$ water, $0.1 \%$ formic acid ( $\mathrm{pH} 2.8$ ) and (b) $100 \%$ water, $25 \mathrm{mM}$ ammonium acetate and $1.25 \%$ ammonium hydroxide $(\mathrm{pH} 9.9)$ in direct infusion mode. Each trace represented an average of 100 scans. No significant decrease in intensities were observed.

Figure S4. Full MS spectrum of A2G2S2 glycopeptide derived from bovine fetuin. Both ammoniated and sodiated adducts were detected due to the additives in mobile phases.

Figure S5. EICs of N156 glycopeptide PGC-LC-MS with 15 injections. The first 10 runs were made back to back and runs 11-15 were made on the second day of experiment. The retention time between each run was less than $1 \mathrm{~min}$, demonstrating the reproducibility of the separation method.

Figure S6. MS/MS spectra of A2G2S2 glycopeptide isomers with (a) both sialic acids with $\alpha 2-6$ linkage and (b) one sialic acid with $\alpha 2-3$ linkage and the other with $\alpha 2-6$ linkage. No significant differences were observed between the two spectra.

Figure S7. EICs of A3G3S0 glycopeptides from (a) N156, (b) N176 and (c) N99 sites in desialylated bovine fetuin. Partial separation of galactose linkage isomers can be obtained (a), while no isomeric separation was observed on the other two glycosylation sites.

Figure S8. EICs of glycopeptides identified from glycosylation sites ${ }^{33} \mathrm{Asn},{ }^{72} \mathrm{Asn}$ and ${ }^{93}$ Asn in AGP sample sequentially digested by trypsin and chymotrypsin.

Figure S9. The results from neuraminidase digestion experiment of AGP glycopeptide ENGTISR with A4G4S4. Only the glycopeptide eluted at 24.9 min in (a) remained after $\alpha 2-3$ neuraminidase treatment, indicating that the peak is fully occupied with $\alpha 2-6$ sialic acids. The other two glycopeptides (26.3 min and $27.1 \mathrm{~min}$ peaks in a) possessed at least one $\alpha 2-3$ linked sialic acid, since both peaks disappeared after the $\alpha 2-3$ enzyme digestion (b). All the three peaks (24.9 $\mathrm{min}, 26.3 \mathrm{~min}$ and $27.1 \mathrm{~min}$ ) are associated with sialylated glycopeptides because none was detected after $\alpha 2-3,6,8,9$ neuraminidase digestion (c). However, no further identification was able to achieve due to the complexity of the sample and lack of enzymes specific to $\alpha 2-6$ linked sialic acids. 
Table S1. Retention times and theirs RSDs of tryptic digested fetuin glycopeptide isomers on LCPDCPLLAPL ${ }^{156}$ NDSR over 15 runs.

\begin{tabular}{|c|c|c|c|c|c|c|}
\hline & \multicolumn{6}{|c|}{ Retention time } \\
\hline & $\begin{array}{l}\text { A } 2 \mathrm{G} 2 \mathrm{~S} 2 \\
\text { Isomer1 }\end{array}$ & $\begin{array}{l}\mathrm{A} 2 \mathrm{G} 2 \mathrm{~S} 2 \\
\text { Isomer2 }\end{array}$ & $\begin{array}{l}\text { A3G3S3 } \\
\text { Isomer1 }\end{array}$ & $\begin{array}{l}\text { A } 3 \mathrm{G} 3 \mathrm{~S} 3 \\
\text { Isomer2 }\end{array}$ & $\begin{array}{l}\text { A3G3S4 } \\
\text { Isomer1 }\end{array}$ & $\begin{array}{c}\text { A3G3S4 } \\
\text { Isomer2 }\end{array}$ \\
\hline Run1 & 40.7 & 42.8 & 41.3 & 44 & 40.6 & 43.2 \\
\hline Run2 & 40.3 & 42.2 & 40.7 & 43.2 & 40 & 42.3 \\
\hline Run3 & 40.1 & 42 & 40.5 & 42.8 & 39.7 & 42 \\
\hline Run4 & 40 & 41.9 & 40.3 & 42.6 & 39.6 & 41.8 \\
\hline Run5 & 39.9 & 41.8 & 40.3 & 42.5 & 39.6 & 41.8 \\
\hline Run6 & 40.1 & 41.9 & 40.3 & 42.5 & 39.6 & 41.8 \\
\hline Run7 & 39.9 & 41.7 & 40.2 & 42.4 & 39.5 & 41.6 \\
\hline Run8 & 39.9 & 41.8 & 40.2 & 42.4 & 39.5 & 41.7 \\
\hline Run9 & 40 & 41.9 & 40.3 & 42.6 & 39.6 & 41.8 \\
\hline Run10 & 40.1 & 42 & 40.4 & 42.7 & 39.7 & 41.9 \\
\hline Run11 & 40.8 & 42.8 & 41.3 & 43.9 & 40.5 & 43.2 \\
\hline Run12 & 40.4 & 42.4 & 40.8 & 43.2 & 40.1 & 42.5 \\
\hline Run13 & 40.4 & 42.3 & 40.7 & 43.1 & 40 & 42.3 \\
\hline Run14 & 40.4 & 42.2 & 40.7 & 43 & 39.9 & 42.2 \\
\hline Run15 & 40.4 & 42.2 & 40.7 & 43.1 & 40 & 42.3 \\
\hline $\begin{array}{c}\text { standard deviation } \\
\text { (Back to Back) (min) }\end{array}$ & 0.2 & 0.3 & 0.3 & 0.5 & 0.3 & 0.5 \\
\hline $\begin{array}{c}\text { relative standard } \\
\text { deviation } \\
\text { (Back-to-Back) }\end{array}$ & $0.6 \%$ & $0.7 \%$ & $0.8 \%$ & $1.2 \%$ & $0.8 \%$ & $1.1 \%$ \\
\hline $\begin{array}{l}\text { standard deviation } \\
\text { (Day to Day) (min) }\end{array}$ & 0.3 & 0.3 & 0.3 & 0.5 & 0.3 & 0.5 \\
\hline $\begin{array}{c}\text { relative standard } \\
\text { deviation } \\
\text { (Day to Day) }\end{array}$ & $0.7 \%$ & $0.8 \%$ & 0.9 & $1.1 \%$ & $0.8 \%$ & $1.2 \%$ \\
\hline
\end{tabular}


Table S2. Comparison of quantitative results of glycopeptides derived from RNaseB between PGC-LC-MS and previous ${ }^{1} \mathrm{H}$ NMR study by Fu et al. ${ }^{1}$

\begin{tabular}{|c|c|c|c|c|}
\hline \multirow{2}{*}{$\begin{array}{c}\text { Peptide } \\
\text { backbone }\end{array}$} & $\begin{array}{c}\text { Glycan } \\
\text { structure }\end{array}$ & $\begin{array}{c}m / z \text { used for extraction } \\
\left(\left[\mathrm{M}+2 \mathrm{H}^{+}\right]^{2+},\left[\mathrm{M}+\mathrm{H}^{+}+\mathrm{NH} 4^{+}\right]^{2+}\right. \\
\left.\text { and }\left[\mathrm{M}+\mathrm{H}^{+}+\mathrm{Na}^{+}\right]^{2+}\right)\end{array}$ & $\begin{array}{c}\text { Relative } \\
\text { quantitative } \\
\text { results by PGC- } \\
\text { LC-MS }\end{array}$ & $\begin{array}{c}\text { Quantitative } \\
\text { estimation } \\
\text { from 1H } \\
\text { NMR study }\end{array}$ \\
\hline \multirow{5}{*}{ NLTK } & Man5 & $846.3593,854.8726,856.9358$ & 45.8 & 57 \\
\cline { 2 - 6 } & Man6 & $927.3857,935.899,937.9622$ & 33.2 & 31 \\
\cline { 2 - 6 } & Man7 & $1008.4121,1016.9254,1018.9886$ & 7.7 & 4 \\
\cline { 2 - 6 } & Man8 & $1089.4385,1097.9518,1100.015$ & 10.6 & 7 \\
\cline { 2 - 6 } & Man9 & $1170.4649,1178.9782,1181.0414$ & 2.7 & 1 \\
\hline
\end{tabular}


Table S3. Summary of the retention times and glycoforms for each glycopeptide isomers derived from AGP glycopeptides.

was digested with trypsin and chymotrypsin. See additional information provided in the method section of the paper.

\begin{tabular}{|c|c|c|c|}
\hline Peptide backbone & Glycan structure & $\begin{array}{l}m / z \text { used for extraction (including protonated, } \\
\text { single sodiated and single ammoniated species) }\end{array}$ & Retention times (min) \\
\hline \multirow{6}{*}{ LVPVPITNATLDQITGK } & A2G2S1 & $1231.9043,1237.5804,1243.2559$ & 43.2 \\
\hline & $\mathrm{A} 2 \mathrm{G} 2 \mathrm{~S} 2$ & $1328.9361,1334.6122,1336.2638$ & $43.0,45.2$ \\
\hline & A3G3S2 & $1450.6469,1456.3229,1461.9984$ & $43.1,44.4$ \\
\hline & A3G3S3 & $1547.6787,1553.3547,1559.0302$ & $42.9,43.7,46.6$ \\
\hline & A3G3S2F1 & $1499.3328,1505.0089,1510.6844$ & 42.5 \\
\hline & A3G3S3F1 & $1596.3646,1602.0407,1607.7162$ & $42.4,44.3$ \\
\hline \multirow{5}{*}{ NEEYNK } & A2G2S1 & $1355.5158,1364.0296,1366.5070$ & $24.3,24.7$ \\
\hline & A2G2S2 & $1501.0635,1509.5773,1512.0547$ & $24.4,26.3$ \\
\hline & A2G2S2F1 & $1574.0924,1582.6062,1585.0837$ & $24.8,25.8$ \\
\hline & A3G3S3 & $1829.1773,1837.6911,1840.1685$ & $25.8,26.3,28.1$ \\
\hline & A3G3S2F1 & $1268.4732,1274.1493,1279.8248$ & 25.2 \\
\hline \multirow{6}{*}{ YFTPNK } & A2G2S2 & $1487.5838,1496.0976,1498.5751$ & 35.6 \\
\hline & A3G3S3 & $1815.6976,1824.2114,1826.6889$ & $35.6,36.3$ \\
\hline & A3G3S3F1 & $1888.7266,1897.2404,1899.7178$ & 35.4 \\
\hline & A4G4S2 & $1852.7160,1861.2298,1863.7073$ & 36.0 \\
\hline & A4G4S3 & $1332.5116,1338.1876,1343.8631$ & $35.8,37.5,39.0$ \\
\hline & A4G4S4 & $1429.5434,1435.2194,1440.8949$ & $36.9,38.0,39.6$ \\
\hline \multirow{6}{*}{ NTTY } & A3G3S3 & $1680.1134,1688.6272,1691.1047$ & $25.9,26.6,28.4$ \\
\hline & A3G3S3F1 & $1753.1424,1761.6562,1764.1336$ & 25.4 \\
\hline & A4G4S3 & $1242.1221,1247.7981,1253.4737$ & $26.6,27.8,28.2,28.6$ \\
\hline & A4G4S4 & $1339.1539,1344.8299,1350.5055$ & $27.6,28.1,29.0$ \\
\hline & A4G4S3F1 & $1290.8081,1296.4841,1302.1596$ & $25.5,27.5$ \\
\hline & A4G4S4F1 & $1387.8399,1393.5159,1399.1914$ & $25.3,27.0,28.1$ \\
\hline
\end{tabular}




\begin{tabular}{|c|c|c|c|}
\hline \multirow{4}{*}{ ENGTISR } & A3G3S3 & $1213.1348,1218.8108,1224.4863$ & $24.2,24.9,25.9$ \\
\cline { 2 - 4 } & A3G3S3F1 & $1261.8207,1267.4968,1273.1723$ & 23.6 \\
\cline { 2 - 4 } & A4G4S4 & $1431.8773,1437.5533,1443.2289$ & $24.9,26.3,27.1$ \\
\cline { 2 - 4 } & A4G4S4F1 & $1480.5633,1486.2393,1491.9148$ & $23.8,25.3,26.3,26.5$ \\
\cline { 2 - 4 } & A4G4S4F2 & $1529.2492,1534.9253,1540.6008$ & $23.8,25.2,25.7$ \\
\hline
\end{tabular}


Zhu et al. Figure S1

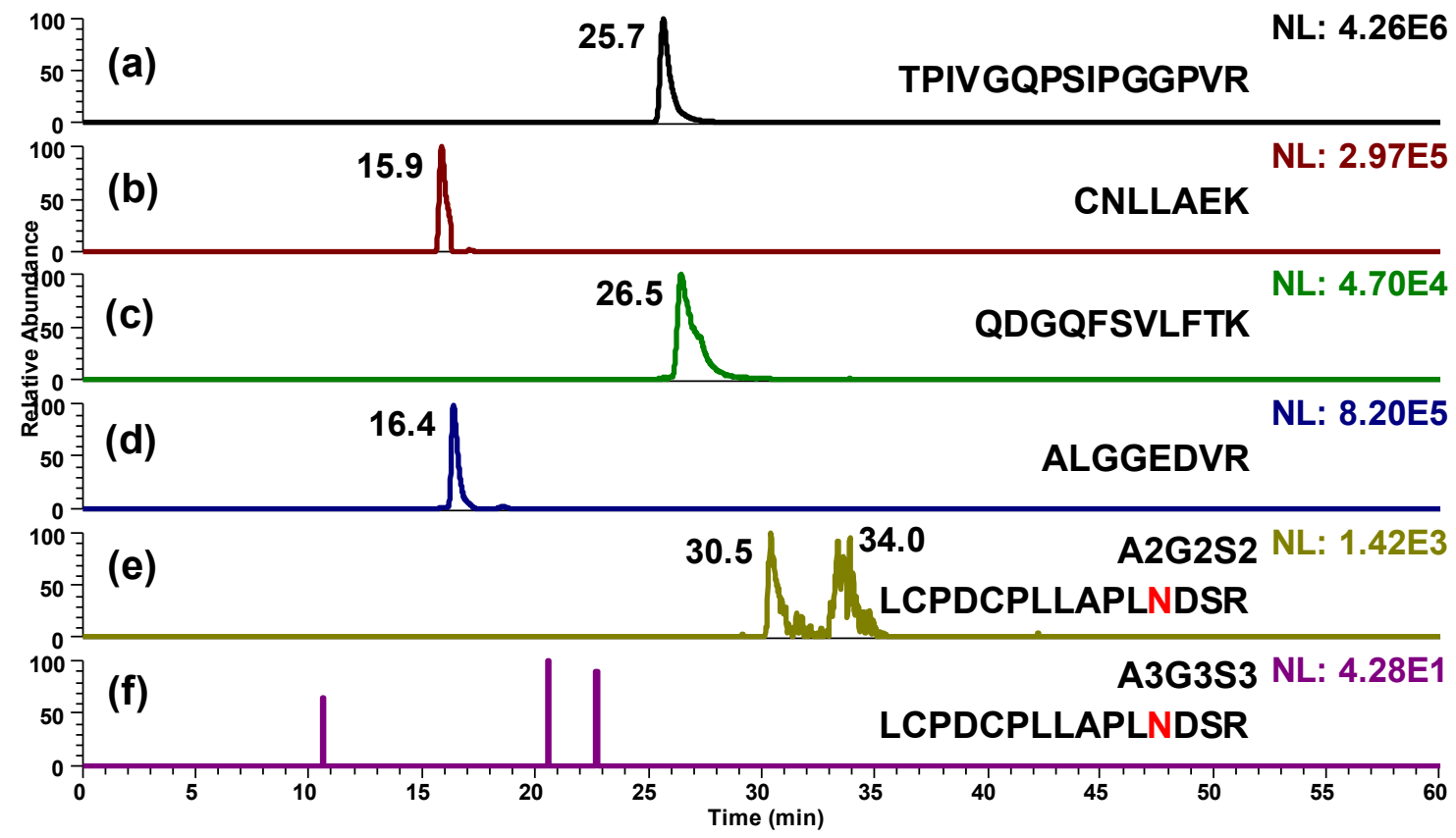

Figure S1. EICs of peptides (a-d) and glycopeptides (e-f) in tryptic digested fetuin sample under acidic conditions. The highly sialylated structures were not eluted from the column. 


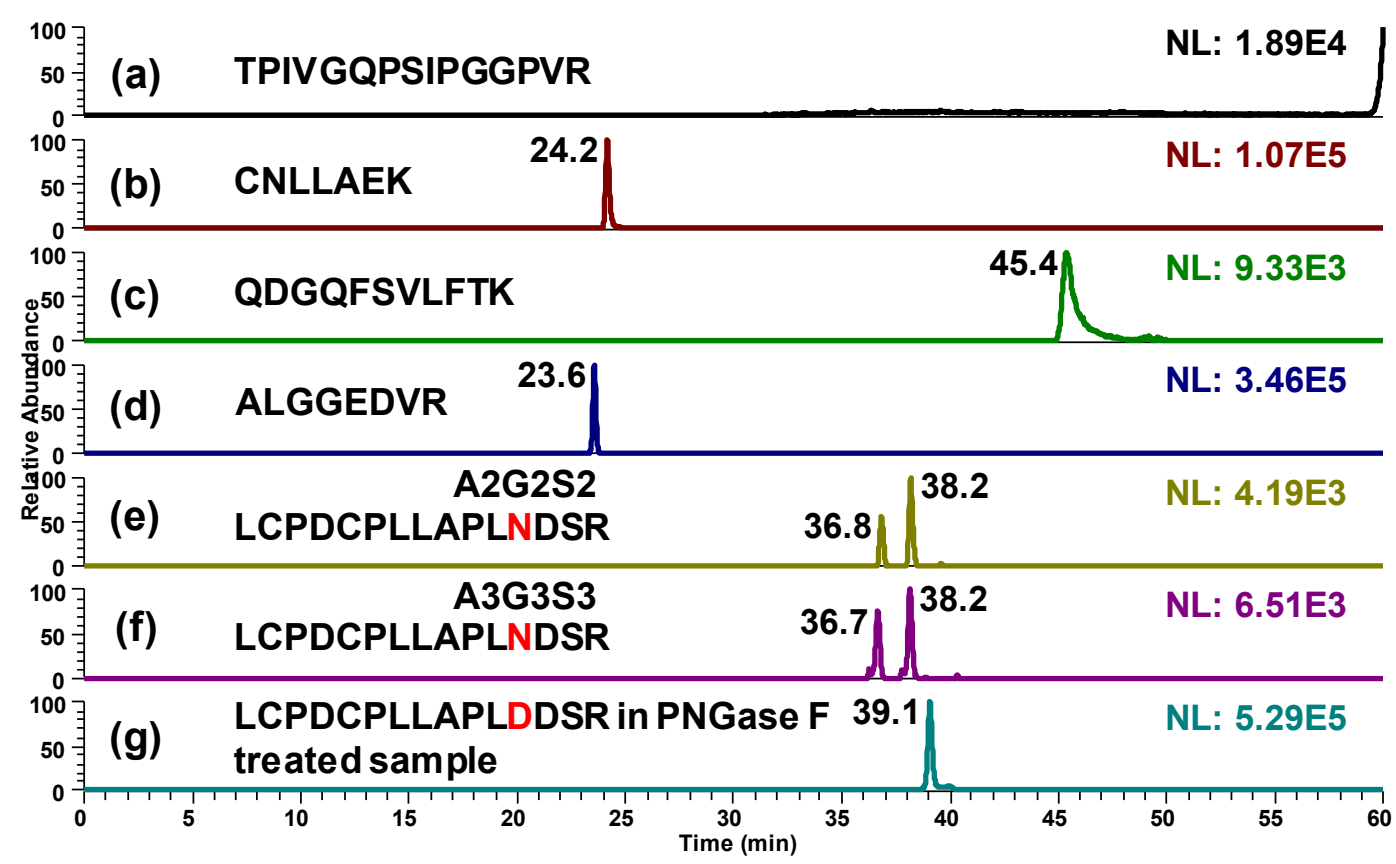

Figure S2. EICs of peptides (a-d) and glycopeptides (e-f) in tryptic digested fetuin sample under basic conditions. Glycopeptides were isomerically separated with stronger retention and uniformed charge states. Deglycosylated peptide backbone were not resolved indicating the separation was prompted by glycan isoforms $(\mathbf{g})$. 
Zhu et al. Figure S3

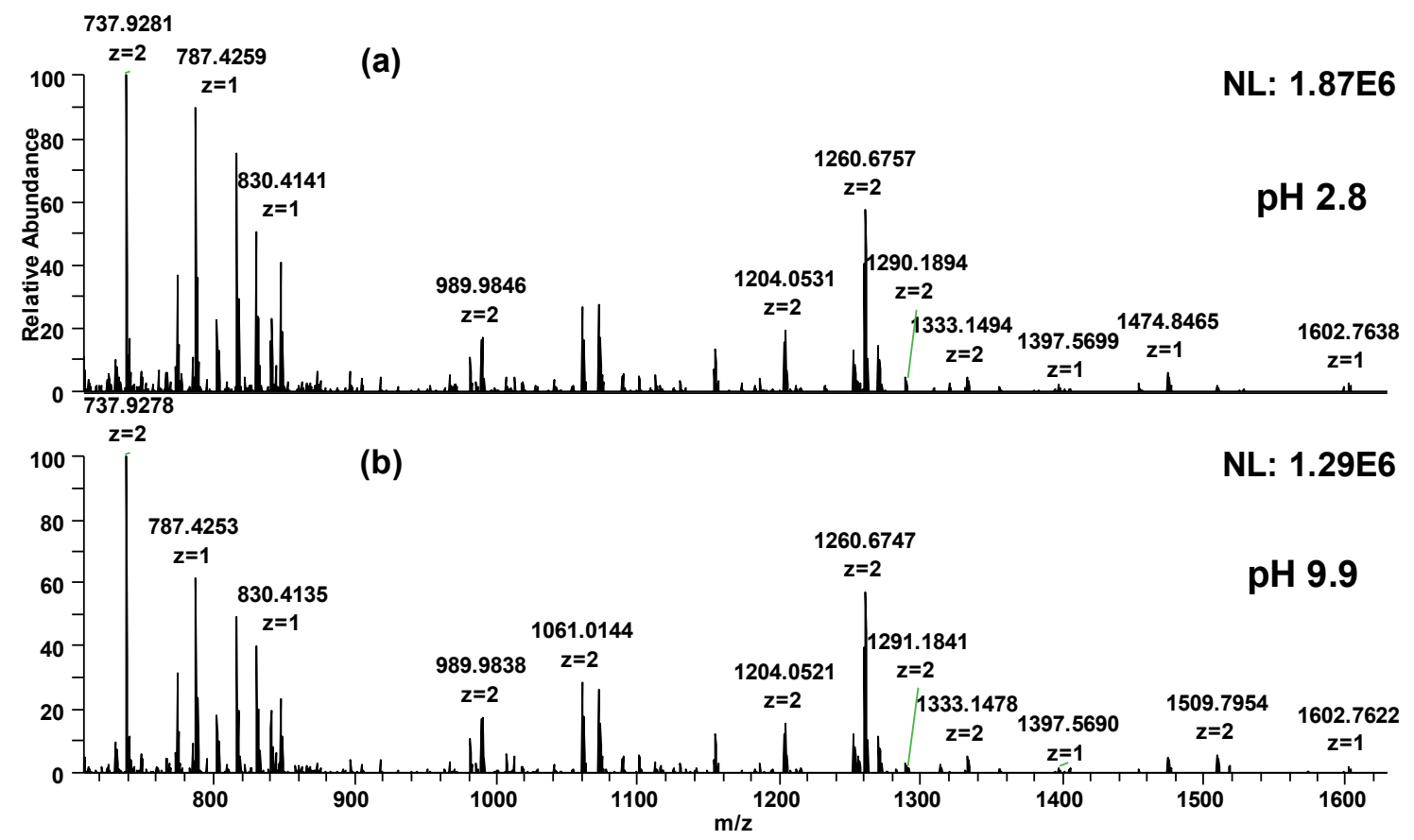

Figure S3. Intensity comparison of tryptic digest of bovine fetuin under (a) $100 \%$ water, $0.1 \%$ formic acid (pH 2.8) and (b) $100 \%$ water, $25 \mathrm{mM}$ ammonium acetate and $1.25 \%$ ammonium hydroxide (pH 9.9) in direct infusion mode. Each trace represented an average of 100 scans. No significant decrease in intensities were observed. 


\section{Zhu et al. Figure S4}

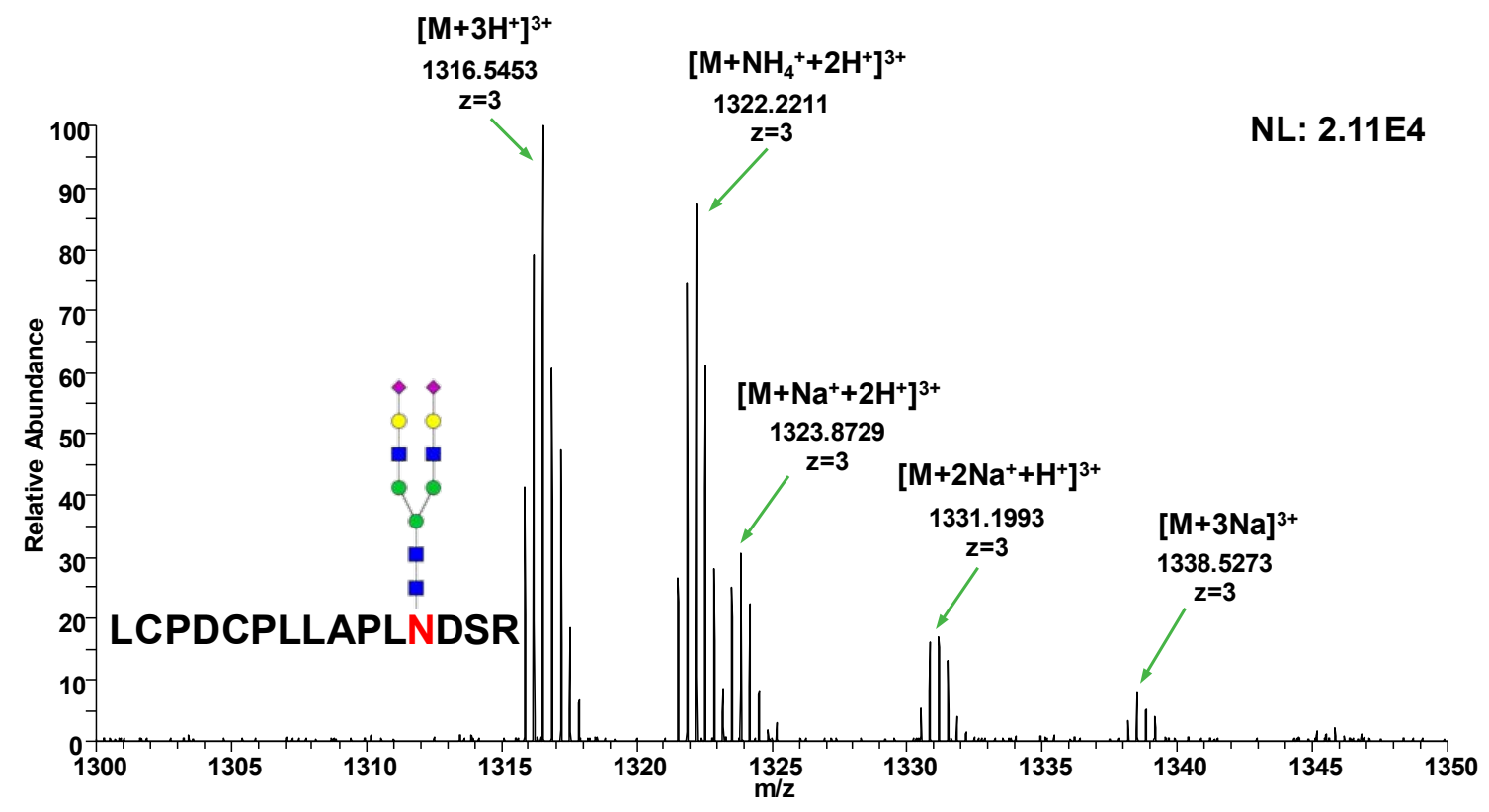

Figure S4. Full MS spectrum of A2G2S2 glycopeptide derived from bovine fetuin.

Both ammoniated and sodiated adducts were detected due to the additives in mobile phases. 
Zhu et al. Figure S5

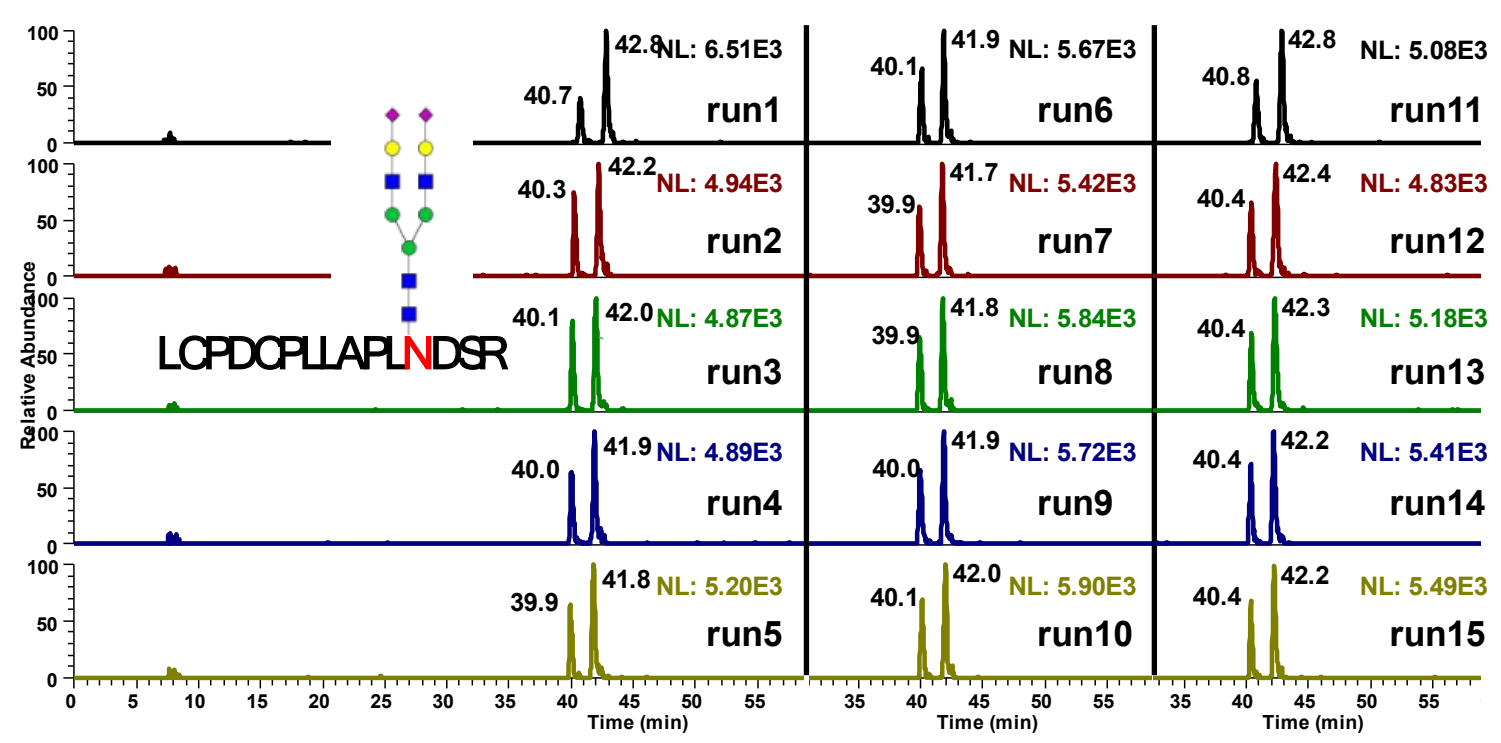

Figure S5. EICs of N156 glycopeptide PGC-LC-MS with 15 injections. The first 10 runs were made back to back and runs 11-15 were made on the second day of experiment. The retention time between each run was less than $1 \mathrm{~min}$, demonstrating the reproducibility of the separation method. 


\section{Zhu et al. Figure S6}

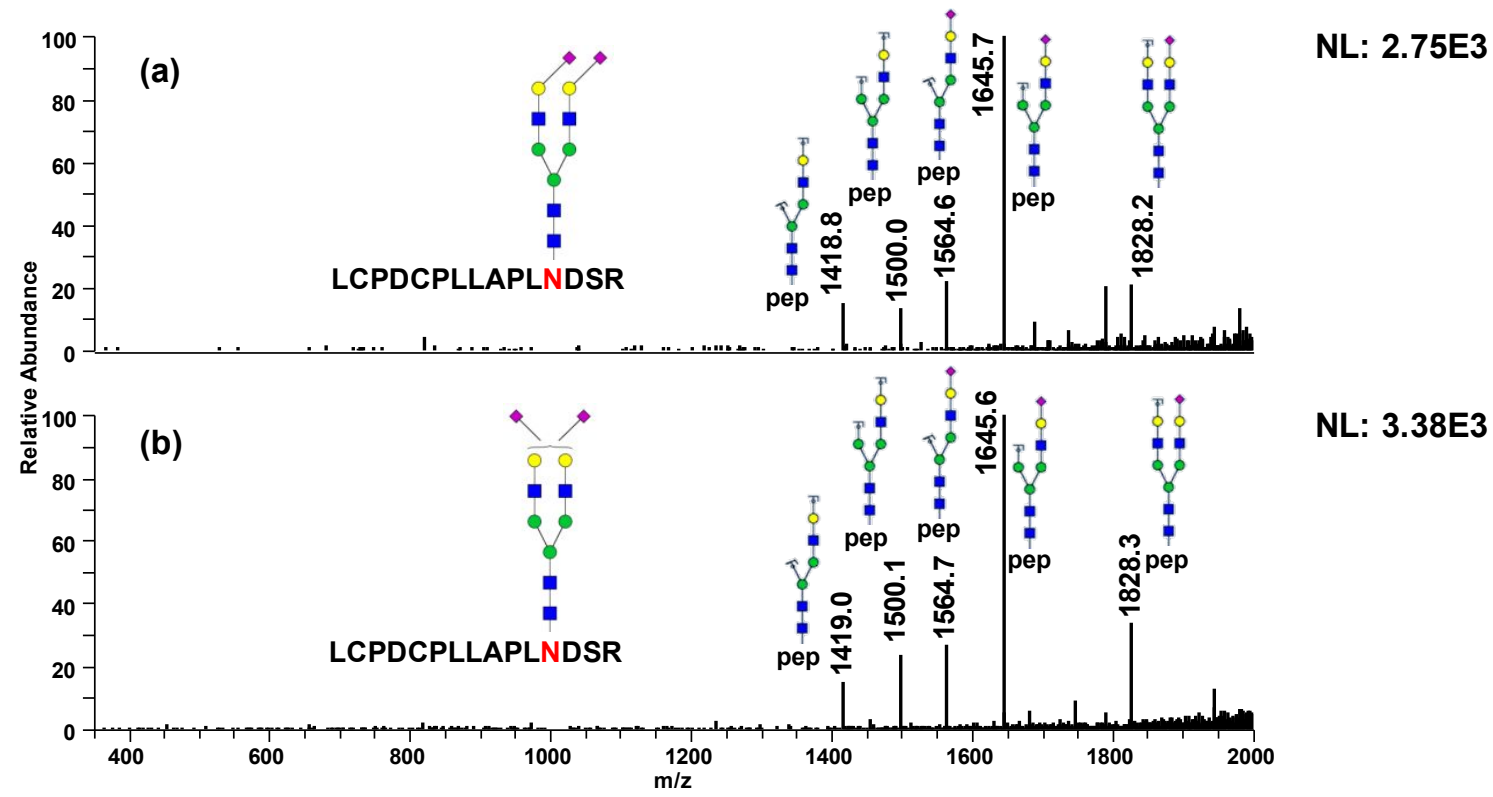

Figure S6. MS/MS spectra of A2G2S2 glycopeptide isomers with (a) both sialic acids with $\alpha 2-6$ linkage and (b) one sialic acid with $\alpha 2-3$ linkage and the other with $\alpha 2-6$ linkage. No significant differences were observed between the two spectra. 


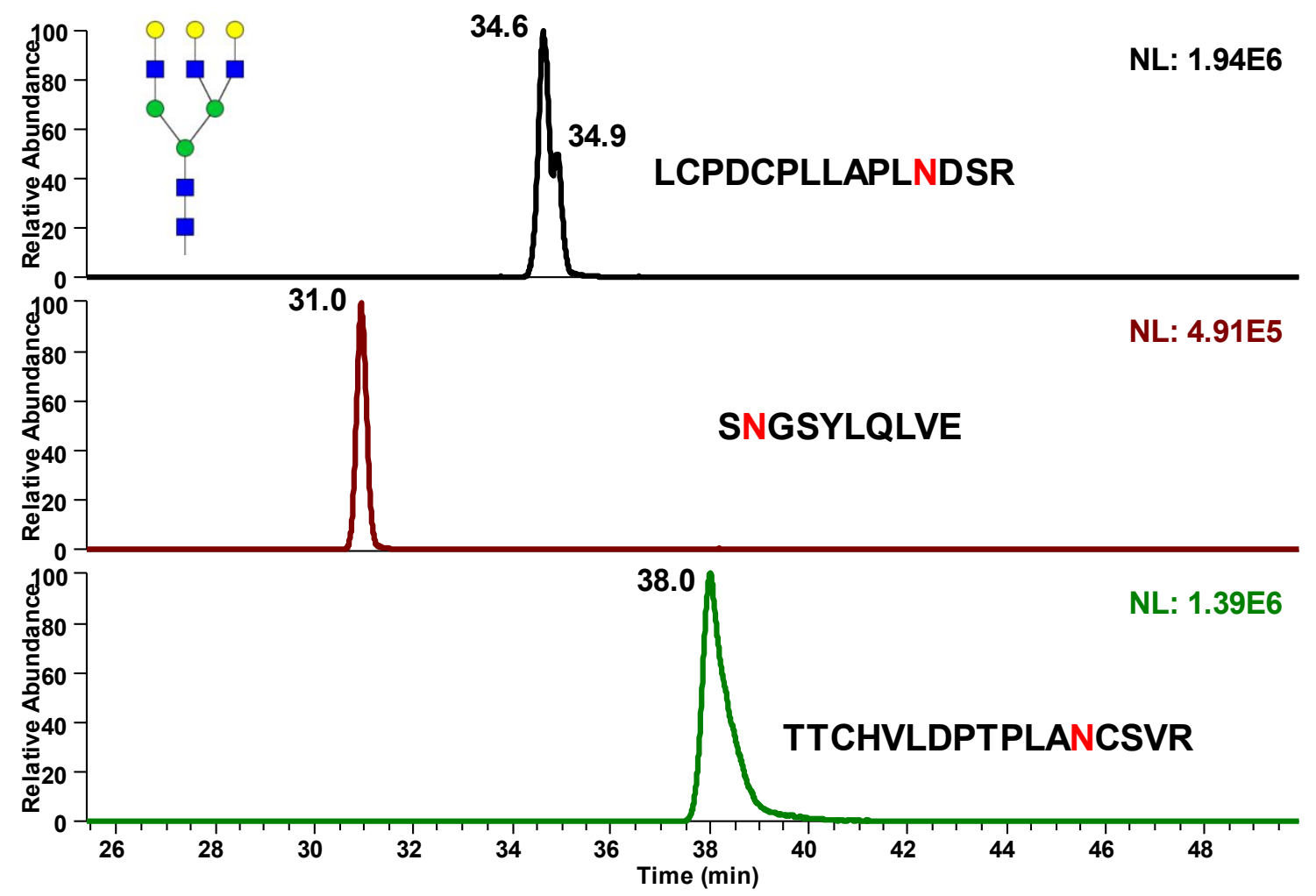

Figure S7. EICs of A3G3 glycopeptides from (a) N156, (b) N176 and (c) N99 sites in desialylated bovine fetuin. Partial separation of galactose linkage isomers can be obtained (a), while no isomeric separation was observed on the other two glycosylation sites. 


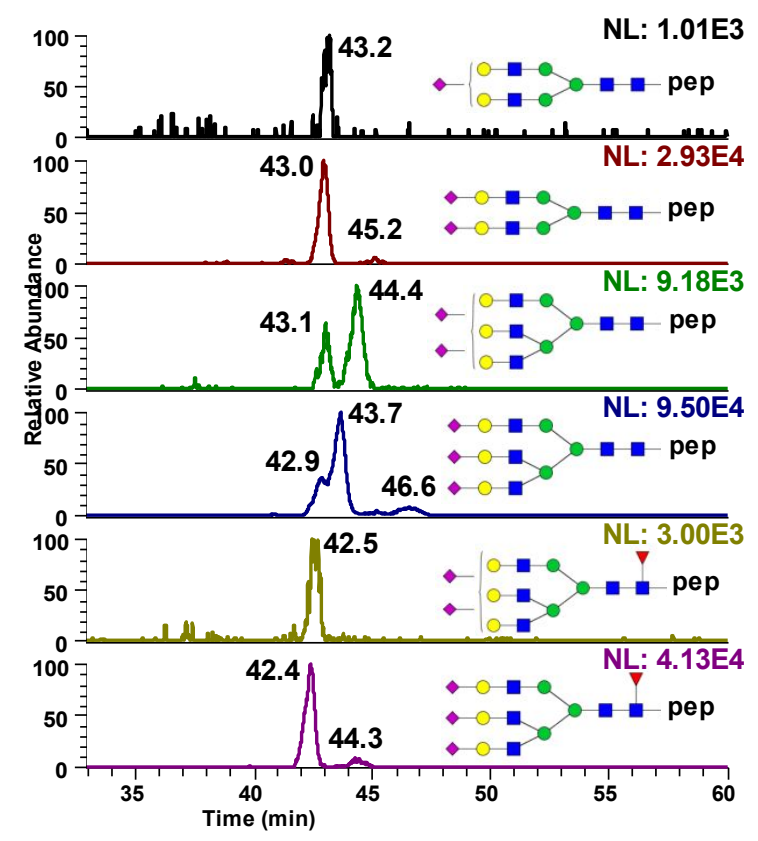

\section{LVPVPIT ${ }^{33}$ NATLDQITGK}

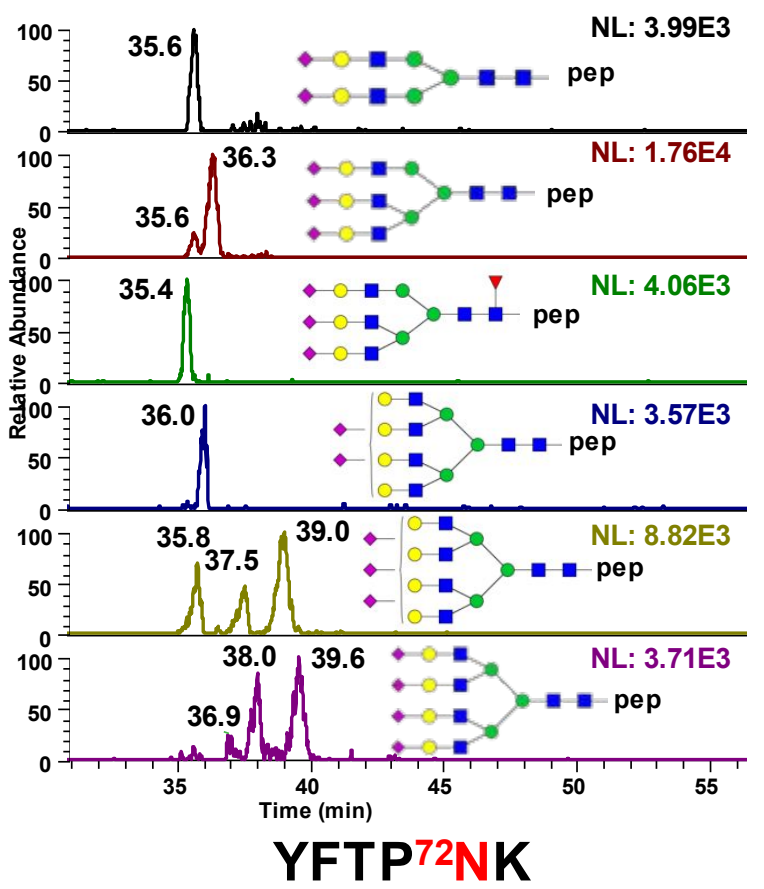

Figure S8. EICs of glycopeptides identified from glycosylation sites ${ }^{33}$ Asn, ${ }^{72}$ Asn and

${ }^{93}$ Asn in AGP sample sequentially digested by trypsin and chymotrypsin. 


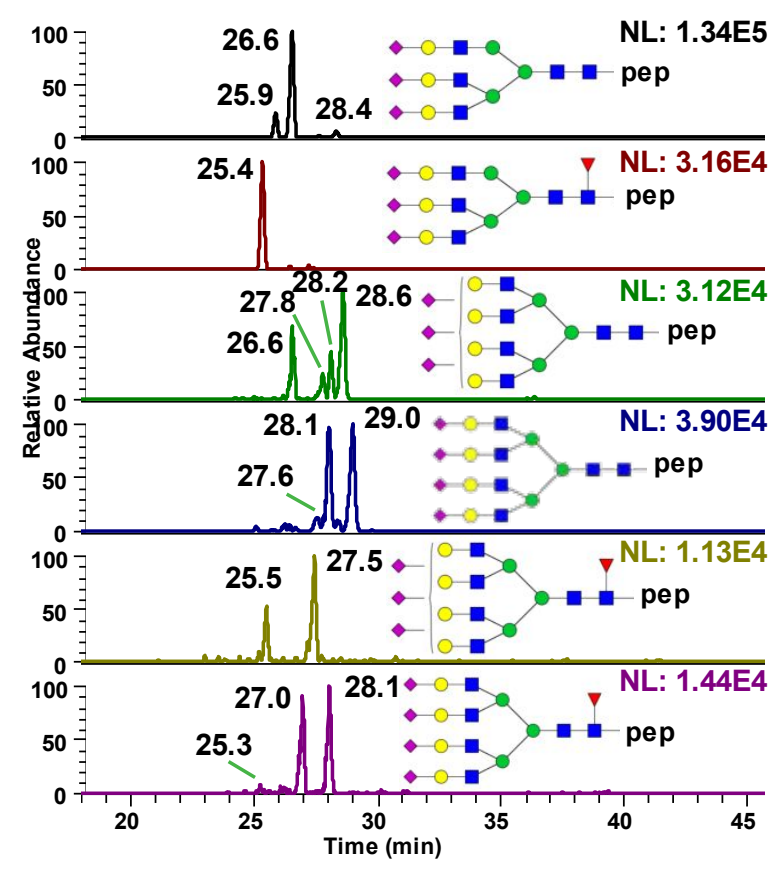

93 NTTY

Figure S8 (cont') EICs of glycopeptides identified from glycosylation sites ${ }^{33}$ Asn,

${ }^{72}$ Asn and ${ }^{93}$ Asn in AGP sample sequentially digested by trypsin and chymotrypsin. 


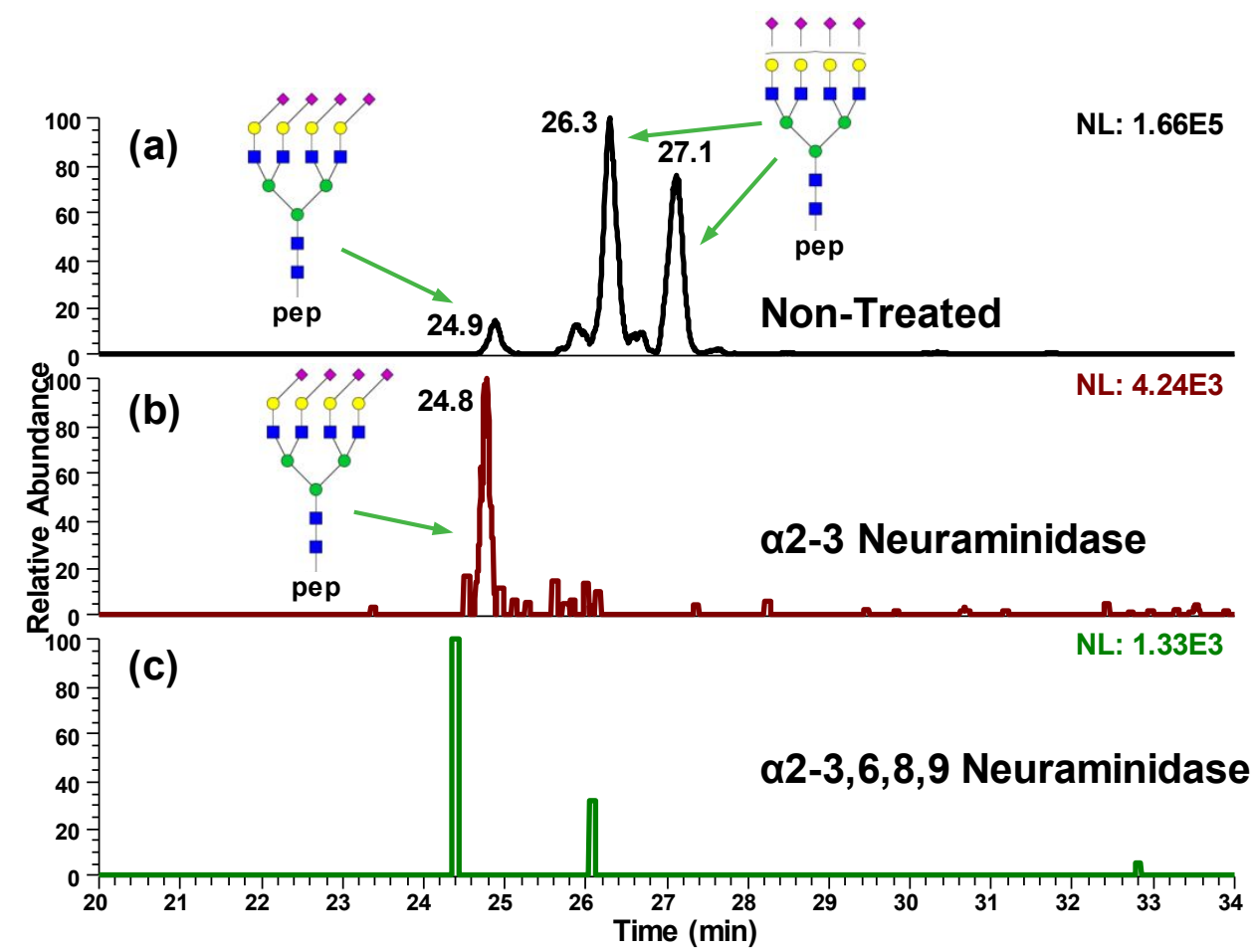

Figure S9. The results from neuraminidase digestion experiment of AGP glycopeptide ENGTISR with A4G4S4. Only the peak at $24.9 \mathrm{~min}$ in (a) remained after $\alpha 2-3$ neuraminidase treatment, indicating that the peak is fully occupied with $\alpha 2-6$ sialic acids. The other two peaks (26.3 min and 27.1 min peaks in a) possessed at least one $\alpha 2-3$ linked sialic acid, since both peaks disappeared after the $\alpha 2-3$ enzyme digestion (b). All the three peaks (24.9 $\mathrm{min}, 26.3 \mathrm{~min}$ and $27.1 \mathrm{~min}$ ) are associated with sialylated glycopeptides because no peaks were detected after $\alpha 2-3,6,8,9$ neuraminidase digestion (c). However, no further identification was able to achieve due to the complexity of the sample and lack of enzymes specific to $\alpha 2-6$ linked sialic acids. 


\section{References}

(1) Fu, D.; Chen, L.; O'Neill, R. A. Carbohydr Res 1994, 261, 173-186. 